\title{
Prevalence of Radiological Abnormalities Among Psychiatric Inpatients: A Study from the United Arab Emirates
}

\author{
Amir Mufaddel ${ }^{1, ~ *}$, Ghanem Al Hassani ${ }^{2}$, Shakhboot Al Bedwawi ${ }^{2}$, Ossama Osman ${ }^{3}$ \\ ${ }^{1}$ Department of Medicine, Psychiatry Ivision, Tawam Hospital, Al Ain, Nited Arab Emirates \\ ${ }^{2} \mathrm{Al}$ Ain Hospital, Al Ain, Nited Arab Emirates \\ ${ }^{3}$ Department of Psychiatry and Behavioural Sciences, College of Medicine and Health Sciences, United Arab Emirates University, Alain, \\ United Arab Emirates
}

Email address:

khalifaamir@yahoo.co.uk (A. Mufaddel)

${ }^{*}$ Corresponding author

\section{To cite this article:}

Amir Mufaddel, Ghanem Al Hassani, Shakhboot Al Bedwawi, Ossama Osman. Prevalence of Radiological Abnormalities Among Psychiatric Inpatients: A Study from the United Arab Emirates. American Journal of Psychiatry and Neuroscience.

Vol. 7, No. 4, 2019, pp. 147-152. doi: 10.11648/j.ajpn.20190704.19

Received: December 5, 2019; Accepted: December 18, 2019; Published: December 30, 2019

\begin{abstract}
Background: This is a cross sectional retrospective study aimed to estimate the prevalence of brain aberrations visible on computed tomography (CT) scan or magnetic resonance imaging (MRI) in psychiatric patients admitted to the main tertiary hospital of Alain city in the United Arab Emirates. Methods: Between January 2011 and December 2013, 1,586 patients were admitted study of patients admitted to the psychiatric ward in Al Ain Hospital. Head CT or brain MRI was requested for $295(19 \%)$ patients. Results: Ninety-four (31.8\%) of the 295 patients had abnormalities on the CT or MRI. The most common finding was calcification, detected in $21(22.3 \%)$ patients. The most common site of calcification was the basal ganglia (15 patients, or 16\%). Multiple site involvements were also common (16 patients, or 17\%). Other types of brain abnormalities included evolutionary changes (dilated ventricles, atrophy and prominent sulci) reported in 13 patients which represent $4.4 \%$ of the total sample and $13.8 \%$ of those with positive findings. Similar number was reported for patients with findings of vascular lesions (ischemic changes / infarction). Basal ganglia was the most common site of reported involvement occurring in 19 subjects and representing $6.4 \%$ of the total sample and $20.2 \%$ of those with positive findings. Common age at presentation with positive findings was 30-39y. Conclusions: Radiological abnormalities are common among psychiatric inpatients. Future studies are needed to identify the potential significance of these findings and correlate them with patient's psychopathology.
\end{abstract}

Keywords: Radiologic Findings, Psychiatric Patients, Brain Sites, Calcifications

\section{Introduction}

Understanding Radiological findings among psychiatric patients are important for more accurate assessment and management of different aspects of psychiatric presentations.

The literature on CT studies in schizophrenia revealed structural changes including enlargement of the ventricles, cerebral sulci and fissure. Similarly, MRI studies had revealed significant differences in ventricular volume and medial temporal structures among these patients [1].
Radiological investigations are used in the workup of patients with psychiatric symptoms in order to rule out possible underlying neurological conditions. Example of lesions that can be detected by neuroimaging are frontal or temporal lobes tumors [2].

Neuroimaging can be also helpful in differentiating presence of a neurodegenerative condition from depression in older people who present with history of cognitive impairment. Example of these are findings on neuroimaging that can be characteristic of Alzheimer's disease, diffuse Lewy body dementia, or frontotemporal dementias. In 
Alzheimer's disease, medial temporal atrophy can be detected by MRI or CT studies [3].

Some studies on the use of brain imaging in first episode psychosis suggest that routine CT or MRI scans are of little benefit and should be reserved for age groups 50 years and older or those with possible neurological problems [4]. Other studies reported about 30\% abnormal findings of previously undiagnosed pathology on MRI scans of psychiatric patients [5]. Moreover; several brain imaging studies have shown structural, functional and neurochemical deviations in subjects who were in phase of At Risk Mental State (ARMS) defined as 5 years prodromal period before the manifestations were clear; with findings similar to those observed when the psychosis is clearly established [6]. The MRI findings in individuals who were at high risk of schizophrenia have shown a significantly higher proportion of radiological findings in individuals who were at high risk of schizophrenia (35\%) as well as those who developed first-episode psychosis (40\%) compared with patients with depression $(18 \%)$ or healthy controls (12\%) [7]. As suggested by some MRI abnormalities, these abnormalities can either precedes the onset of psychosis as suggested by the neurodevelopmental models of schizophrenia or may develop progressively through the illness course [8].
The current study is a cross-sectional retrospective study aimed to estimate the prevalence of brain abnormalities visible on computed tomography (CT) scan or magnetic resonance imaging (MRI) in psychiatric patients admitted to Alain Hospital. The latter is the main tertiary hospital with multiple specialized facilities including a psychiatric ward with a 32 psychiatric inpatient beds located in the city of Alain, in the Gulf country of the United Arab Emirates. Alain hospital is the only psychiatric emergency receiving facility for that region with catchment area of around 750.000 population. It has an Electronic Health record system which was established in 2010. The electronic database of all patients admitted between January 2011 and December 2013 were reviewed and records of patients subjected to brain imaging were retrieved. Patients with previous radiological investigations were identified for inclusion into the study, and were given serial numbers for anonymity. Patients with multiple admissions were identified and were given only one serial number each regardless of the number of admissions. Figure 1 shows the sample selection procedure whereby a data collection form was designed for recording data of patients with both positive and negative radiological results. The form included the socio-demographic information, the clinical psychiatric presentation and the medical history of each patient.

\section{Methods}

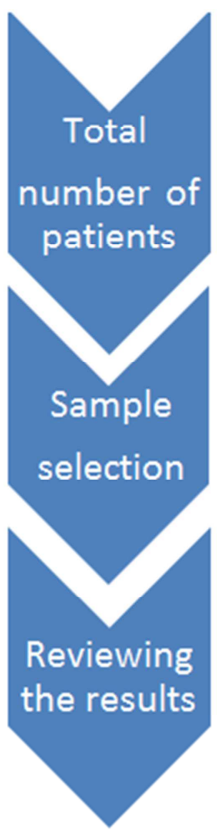

- Data source included a total of 1586 patients admitted between 2011 \& 2013.

- Records were reviewed for presence of radiological studies. A total of 295 radiological results were obtained.

-A serial number was given for each patient with radiological studies.

- Number of patients with positive radiological results: 94

- Number of patients with negativr radiological results: 201

Figure 1. Selection procedure of the study sample.

We compared the group's positive and negative results accordingly. Data were analyzed using the SPSS version 21. We excluded from the analysis any uncompleted, uncertain or missing data. When we presented the results of our study, we indicated the number of sample with completed data in each of the tables and Chi-square was used to assess significance which was set at 0.05 .

\section{Results}

\subsection{Socio-demographic and Psychiatric Characteristics}

A total 1586 patients were admitted to the psychiatric ward during the study period. Table 1 shows the Socio- 
demographic and psychiatric characteristics of the of the entire study sample. Radiological investigations were ordered for 295 of these patients. Only 94 subjects had abnormal findings which represent $32 \%$ of those patients subjected to radiological examination.

Table 1. Socio-demographic and clinical psychiatric characteristics.

\begin{tabular}{|c|c|c|c|}
\hline Variable & Positive radiological findings & Negative Radiological findings & Statistical significance \\
\hline \multicolumn{4}{|l|}{ Age groups $(\mathrm{N}=282)^{*}$} \\
\hline Less than 20 & 03 & 18 & \multirow{7}{*}{$\mathrm{P}<0.0001$} \\
\hline 20 to 29 & 25 & 87 & \\
\hline 30 to 39 & 28 & 48 & \\
\hline 40 to 49 & 19 & 22 & \\
\hline 50 to 59 & 07 & 11 & \\
\hline Over 60 & 12 & 02 & \\
\hline Total & 94 & 188 & \\
\hline \multicolumn{4}{|l|}{ Gender $(\mathrm{N}=282)^{*}$} \\
\hline Male & 64 & 125 & \multirow{3}{*}{$\mathrm{p}=0.449$} \\
\hline Female & 30 & 63 & \\
\hline Total & 94 & 188 & \\
\hline \multicolumn{4}{|c|}{ Psychiatric diagnosis $(\mathrm{N}=265)^{*}$} \\
\hline Mood disorder & 39 & 12 & \multirow{7}{*}{$\mathrm{P}<0.0001$} \\
\hline Schizophrenia & 15 & 36 & \\
\hline Unspecified psychosis & 10 & 17 & \\
\hline Dementia & 3 & 0.0 & \\
\hline Personality disorder & 5 & 7 & \\
\hline Others & 14 & 89 & \\
\hline Total & 94 & 171 & \\
\hline \multicolumn{4}{|c|}{ Number of psychiatric Episodes $(\mathrm{N}=245)$} \\
\hline One & 37 & 77 & \multirow{4}{*}{$\mathrm{P}=0.039$} \\
\hline Two & 12 & 29 & \\
\hline Three or more & 43 & 47 & \\
\hline Total & 92 & 153 & \\
\hline \multicolumn{4}{|c|}{ Duration since the first Episode $(\mathrm{N}=208)^{*}$} \\
\hline$<$ One year & 29 & 25 & \multirow{5}{*}{$\mathrm{P}<0.0001$} \\
\hline $1-2$ years & 6 & 27 & \\
\hline 3-4 years & 7 & 50 & \\
\hline 5 years or more & 47 & 17 & \\
\hline Total & 89 & 119 & \\
\hline
\end{tabular}

*indicates number of cases valid for analysis.

As shown in table 1, we compared the positive and negative radiologic finding groups in regards to age and gender which revealed positive statistical significance for age $(\mathrm{P}<0.0001)$ but not for gender. There were statistically significant differences between the two groups when compared for the type of psychiatric diagnosis $(p<0.0001)$, number of psychiatric episodes $(p=0.039)$ and duration of psychiatric illness $(\mathrm{P}<0.0001)$.

Based on the documented clinical diagnosis; mood disorders were more common in patients with positive radiological findings, as they were reported in 39 patients (41.1\%) compared with only 12 patients $(4.5 \%)$ of those with negative radiological findings. On the other hand, Schizophrenia was the most common diagnosis among inpatients with negative results, and was reported in 36 patients $(13.5 \%)$. Interestingly, the patients with positive radiological findings had more tendency to have a chronic psychiatric symptoms lasting for 5 or more years $(52.8 \%)$, compared with $14.2 \%$ with similar duration among those who had negative findings.

\subsection{Types of Reported Radiologic Abnormalities}

Table 2 shows the different types of reported radiological abnormalities. Cerebral calcifications was the most reported type and was found in 21 subjects representing $22.3 \%$ of the group with positive findings and $7.1 \%$ of overall patients. The most common site of calcification was reported in the basal ganglia. The later was studied separately compared with controls and the results of the study were previously published [9]. Calcification were also reported in falx cerebri, pineal gland and calcified frontal lobe meningioma. Other types of reported brain aberrations included evolutionary changes (dilated ventricles, atrophy and prominent sulci) existing in 13 patients which represent $4.4 \%$ of the total sample and $13.8 \%$ of those with positive findings. Similar number was reported for patients with findings of vascular lesions (ischemic changes / infarction).

Table 2. Types of lesions.

\begin{tabular}{llll}
\hline Type & Number of cases & \% Positive group (N=94) & $\begin{array}{l}\text { \% Total of positive and negative radiological } \\
\text { findings (N=295) }\end{array}$ \\
\hline Calcification & 21 & 22.3 & 7.1 \\
\hline
\end{tabular}




\begin{tabular}{llll}
\hline Type & Number of cases & \% Positive group (N= 94) & $\begin{array}{l}\text { \% Total of positive and negative radiological } \\
\text { findings (N=295) }\end{array}$ \\
\hline Atrophy/ dilated ventricles/ prominent sulci & 13 & 13.8 & 4.4 \\
Vascular/ Infarction/ ischemic & 10 & 10.6 & 3.4 \\
Nonspecific hypo-or hyperdense lesions & 13 & 13.8 & 4.4 \\
Lesions involving the skull or sinuses & 13 & 13.8 & 4.4 \\
Gliosis/ surgery/ craniotomy & 05 & 05.3 & 1.7 \\
Multiple lesions & 05 & 05.3 & 1.7 \\
Others & 14 & 14.9 & 4.7 \\
\hline
\end{tabular}

\subsection{Sites of Reported Lesions}

Table 3 displays the reported Sites of lesions. The Basal ganglia was the most reported site of involvement occurring in 19 subjects and representing $6.4 \%$ of the total sample and $20.2 \%$ of those with positive findings. Lesions of basal ganglia other than calcifications was reported in only 4 patients. We found that multiple sites involvement was also common, occurring in 16 patients which represent $17 \%$ of the group with positive sample and $5.4 \%$ of the total sample.

Table 3. Sites of lesions.

\begin{tabular}{llll}
\hline Site & Number of cases & $\begin{array}{l}\text { \% Subjects with Positive Radiologic } \\
\text { findings (N=94) }\end{array}$ & $\begin{array}{l}\text { Total Subjects with radiological studies } \\
\text { (N=94) }\end{array}$ \\
\hline Basal ganglia & 19 & 20.21 & 6.44 \\
Multiple involvement & 16 & 17.02 & 5.42 \\
Skull and sinuses & 16 & 17.02 & 5.42 \\
Others & 12 & 12.76 & 4.07 \\
Frontal lobe & 8 & 08.51 & 2.71 \\
Temporal lobe & 4 & 04.25 & 1.36 \\
Ventricular/periventricular & 4 & 04.25 & 1.36 \\
Cisterns & 4 & 04.25 & 1.36 \\
Parietal lobe & 3 & 03.19 & 1.02 \\
Occipital lobe & 2 & 02.13 & 0.68 \\
Cortex & 2 & 02.13 & 0.68 \\
Meninges & 2 & 02.13 & 0.68 \\
Cerebellum & 2 & 02.13 & 0.68 \\
\hline
\end{tabular}

Frontal lobe lesions occurred in $8.5 \%$ of those with positive findings and $2.7 \%$ of the total sample. Temporal lobe, ventricular and periventricular areas, and cisterns had similar percentage each representing $4.2 \%$ of the positive group and $1.2 \%$ of the total sample. Occipital lobe, cerebellum, cortex and meninges were less common and each has been reported in $2.1 \%$ of the positive group and $0.6 \%$ of the total sample.

\subsection{Medical History}

Chronic medical conditions were reported in 50 out of 94 patients with positive radiological findings (53.2\%) compared with 35 out of 154 control subjects (22.7\%) which has high statistical significance $(\mathrm{P}<0.0001)$. The majority of patients with positive findings suffered from multiple medical conditions $(25.5 \%)$ followed by neurological $(8.5 \%)$, cardiac diseases $(7.5 \%)$ and endocrine disorder $(6.4 \%)$.

Reporting physical symptoms upon admission to the psychiatric ward was also common among those who had positive findings (46.8\%) compared with only $3.7 \%$ of the control group $(\mathrm{P}<0.0001)$.

Positive history of seizure was statistically significant finding that was reported in $25.5 \%$ of cases with positive findings and $15.6 \%$ of the control subjects $(\mathrm{P}=0.039)$.

Table 4. Medical History in patients with positive findings compared with controls.

\begin{tabular}{llll}
\hline & $\begin{array}{l}\text { Cases with positive findings } \\
\mathbf{N}(\%)\end{array}$ & $\begin{array}{l}\text { (Control) Cases with positive } \\
\text { findings N (\%) }\end{array}$ & Significance \\
\hline Chronic medical conditions $(\mathrm{N}=248)^{*}$ & & & \\
Endocrine & $06(6.4)$ & $08(5.2)$ & $12(7.8)$ \\
Neurological & $08(8.5)$ & $03(1.9)$ & $\mathrm{P}<0.0001$ \\
Cardiac & $07(7.5)$ & $11(7.1)$ & \\
Other & $05(5.3)$ & $0.0(0.0)$ & $119(77.3)$ \\
Multiple medical conditions & $24(25.5)$ & $154(100)$ & $\mathrm{P}<0.0001$ \\
No history of chronic medical conditions & $44(46.8)$ & & \\
Total & $94(100)$ & $06(3.7)$ & $157(96.3)$ \\
Presence of physical symptoms on admission: $(N=257)^{*}$ & & $163(100)$ & \\
Present & $44(46.8)$ & $50(53.2)$ & \\
Not present & $94(100)$ &
\end{tabular}




\begin{tabular}{llll}
\hline & $\begin{array}{l}\text { Cases with positive findings } \\
\text { N (\%) }\end{array}$ & $\begin{array}{l}\text { (Control) Cases with positive } \\
\text { findings N (\%) }\end{array}$ & Significance \\
\hline History of seizure $(\mathrm{N}=261)^{*}$ & & & \\
Positive history of seizures & $24(25.5)$ & $26(15.6)$ & $\mathrm{P}=0.037$ \\
Negative history of seizures & $70(74.5)$ & $141(84.4)$ & \\
Total & $94(100)$ & $167(100)$ & \\
\hline
\end{tabular}

${ }^{*}$ indicates number of cases valid for analysis.

\section{Discussion}

Structural brain imaging (CT scans and MRI) may be requested by psychiatrists in order to identify possible underlying organic etiology particularly for young patients and those who present at first-episode psychosis. Possible findings as suggested in the literature include brain injury, demyelination, brain tumors, multiple sclerosis or stroke [10]. Radiological investigations are frequently ordered for psychiatric patients. In one study, CT-head scans were ordered for $44.4 \%$ of patients with bipolar disorder, $23 \%$ of patients with dissociative disorder, and $21.6 \%$ of those with depressive disorder [11]. However, the radiologists may have different opinions and criticism regarding the radiological investigations for psychiatric patients and what to look for in such group of patients. In the past, the radiologists were concerned with reviewing findings to exclude any structural abnormality that might explain the psychiatric presenting symptoms such as temporal lobe abnormalities. Most of the time, radiological images were interpreted as normal, and were not helpful in work-up of psychiatric symptoms [12].

Therefore, retrospective study of the results obtained from the radiological examinations that were ordered based on clinical impression is essential as these data are accumulating over time and assumed to be ordered because of specific cause that made the presence of organic background more likely for the clinician who initially assessed the psychiatric symptoms. In some cases, routine radiological examination can lead to identification of rare of multi-system involvement [13]. If the psychiatric symptoms can be explained pathologically by an underlying disease process, the diagnosis is most likely to be a psychiatric disorder due to a medical condition, rather than primary/functional psychiatric illness. So, itis important to differentiate psychiatric symptoms directly related to organic causes from those coincidentally occurring in a patient suffering from a primary psychiatric disorder [14].

Our findings suggest that nearly one third of the results of radiological examinations have shown some sort of abnormal finding. These findings may not necessarily have direct explanation of presenting symptoms in a way that it can change the diagnosis or modify treatment plan. For example, presence of dilated ventricles or calcified brain lesions is not a direct explanation suggesting another diagnosis or alternative treatment plan for patients presenting with psychiatric symptoms such as schizophrenia or mood disorder. However, presenting the findings of radiological examinations can lead to further research aiming to give possible pathological explanation in the future, particularly when such findings are associated with risk factors such as age, psychiatric presentation, duration of symptoms, number of episodes and presence of associated medical conditions that were all found to be statistically significant in our study. Follow -up radiological studies may be necessary in some cases as the radiological abnormalities may be progressive over time. In a recent review of patients with recurrent episodes of psychiatric illness, the brain abnormalities were not static but progressive showing extended changes with bilateral gray matter loss in different brain areas including the prefrontal cortex, hippocampus, amygdala, and basal ganglia [12].

The most common type of brain lesions in our study is calcification that was mostly present in basal ganglia. Other sites of calcifications include falx cerebri, pineal gland and calcified frontal lobe meningioma. Calcifications can suggest several differential diagnoses based on other clinical findings. Possible causes include tumors, vascular abnormalities, endocrine causes, infections and genetic factors [15]. The later finding together with presence of high medical comorbidity reported study population makes the possibility of systemic etiology more likely. This may also indicate that the radiological abnormality may appear not specific per se but if correlated with other risk factors can lead to identifying other diagnoses. Structural abnormalities on CT scans in people with schizophrenia include enlargement of the ventricles, cerebral sulci and fissure; and MRI studies confirm significant differences in ventricular volume and medial temporal structures [4].

\section{Conclusion}

Radiological abnormalities are common among psychiatric inpatients. Calcification and atrophic changes are the most common types of abnormality; and the most common sites of abnormality are the basal ganglia followed by abnormalities involving multiple sites. Future studies are needed to identify the potential significance of these findings and correlate them with patient's psychopathology.

\section{Acknowledgements}

All authors have declared no conflict of interest in relation to this research.

This study was conducted without any external funding.

\section{References}

[1] Abiodun OA. Role of radiology in psychiatry: a review. East Afr Med J. 2005; 82 (5): 260-6. 
[2] Bunevicius A, Deltuva VP, Deltuviene D, Tamasauskas A, Bunevicius R. Brain lesions manifesting as psychiatric disorders: eight cases. CNS Spectrum. 2008; 13: 950-958.

[3] Masdeu JC. Neuroimaging in psychiatric disorders. Neurotherapeutics. 2011: 8 (1): 93-102. doi: 10.1007/s13311010-0006-0. PMID: 21274689; PMCID: PMC3052989.

[4] Goulet K, Deschamps B, Evoy F, Trudel J-F. Use of brain imaging (computed tomography and magnetic resonance imaging) in first-episode psychosis: review and retrospective study. Canadian Journal of Psychiatry. 2009; 54: 493-501.

[5] Lubman DI, Velakoulis D, McGorry PD, Smith DJ, Brewer W, Stuart G, Desmond P, Tress B, Pantelis C. Incidental radiological findings on brain magnetic resonance imaging were also reported in first-episode psychosis and chronic schizophrenia. Acta Psychiatrica Scandinavica. 2002; 106: 331-336.

[6] McGuire P. Brain imaging and transition to psychosis. Encephale. 2010; 36 Suppl 3: S66-S70.

[7] S J Borgwardt, E-W Radue, K Götz, J Aston, M Drewe, U Gschwandtner, S Haller, M Pflüger, R-D Stieglitz, P K McGuire, and A Riecher-Rössler. Radiological findings in individuals at high risk of psychosis. J Neurol Neurosurg Psychiatry. 2006; 77 (2): 229-233.

[8] Pantelis C, Velakoulis D, McGorry P D. et al Neuroanatomical abnormalities before and after onset of psychosis: a cross-sectional and longitudinal MRI comparison. Lancet 2003361281-288.
[9] Mufaddel A A, Osman OT, Al-Hassani G, Al-Bedwawi S, Hashim MJ. Basal Ganglia Calcification in Psychiatric Inpatients: A Case-Control Study. Cogn Behav Neurol. 2016 Sep; 29 (3): 139-43.

[10] Ricardo Coentre, Amilcar Silva-dos-Santos, Miguel Cotrim Talina. Retrospective study on structural neuroimaging in first-episode psychosis. PeerJ. 2016; 4: e2069.

[11] Gupta DK, Suthar N, Singh V, et al. Frequency and pattern of radiological and laboratory investigations in patients with mental illnesses: A study from North Rajasthan. Indian J Psychiatry 2016; 58 (2): 183-9.

[12] Agarwal N, John D, Bazzocchi M, Renshaw PF. Update on the Use of MR for Assessment and Diagnosis of Psychiatric Diseases. Radiology 2010; 255: 1 (23-40).

[13] Mufaddel A, AlSabousi M, Saleh B, Alhassani G, Osman OT. A Case of Gorlin-Goltz Syndrome Presented with Psychiatric Features. Behavioural Neurology. 2014; 10.3233/BEN130355 .

[14] Mufaddel A, Omer AA, Salem MO. Psychiatric Aspects of Infectious Diseases. OJP 2014， 4, 202-217. doi: 10.4236/ojpsych.2014.43027.

[15] Mufaddel A, Alhassani G. Familial Idiopathic basal ganglia calcification (Fahr's disease), Neurosciences, 2014: 19 (3), 171. 